\title{
Gastric carcinogenesis: a comprehensive review of the angiogenic pathways
}

\author{
Alicja Forma ${ }^{1}\left(\right.$ Magdalena Tyczyńska $^{2} \cdot$ Paweł Kędzierawski $^{1} \cdot$ Klaudyna Gietka $^{1} \cdot$ Monika Sitarz $^{3}$
}

Received: 15 September 2020 / Accepted: 31 October 2020 / Published online: 18 November 2020

(c) The Author(s) 2020

\begin{abstract}
Gastric cancer (GC) is undoubtedly one of the most prevalent malignancies worldwide. Since GC is the second leading cause of cancer-related deaths with nearly one million new diagnoses reported every year, there is a need for the development of new, effective treatment strategies of GC. Gastric carcinogenesis is a complex process that is induced by numerous factors and further stimulated by many pro-oncogenic pathways. Angiogenesis is the process of the new blood vessels formation from the already existing ones and it significantly contributes to the progression of gastric tumorigenesis and the growth of the cancerous tissues. The newly formed vessels provide cancer cells with proper nutrition, growth factors, and oxygen supply that are crucial for tumor growth and progression. Tumor-associated vessels differ from the physiological ones both morphologically and functionally. They are usually inefficient and unevenly distributed due to structural transformations. Thus, the development of the angiogenesis inhibitors that possess therapeutic effects has been the main focus of recent studies. Angiogenesis inhibitors mostly affect the vascular endothelial growth factor (VEGF) pathway since it is a major factor that stimulates the pro-angiogenic pathways. The aim of this review was to describe and summarize other promising molecular pathways that might be crucial in further improvements in GC therapies. This article provides an overview of how a meaningful role in tumor progression the angiogenetic process has. Furthermore, this review includes a description of the most important angiogenic factors as well as pathways and their involvement in gastric carcinogenesis.
\end{abstract}

Keywords Gastric cancer · Carcinogenesis · Tumorigenesis · Angiogenesis · Pro-angiogenic pathways

\begin{tabular}{|c|c|c|c|}
\hline \multicolumn{2}{|c|}{ Abbreviations } & \multirow[t]{2}{*}{ CEACAM6 } & \multirow{2}{*}{$\begin{array}{l}\text { Carcinoembryonic antigen-related cell } \\
\text { adhesion molecule } 6\end{array}$} \\
\hline $\mathrm{AF}$ & $\alpha$-Fetoprotein & & \\
\hline \multirow{3}{*}{\multicolumn{2}{|c|}{ Angiopoietins }} & $\mathrm{COX}-2$ & Cyclooxygenase-2 \\
\hline & & CRYAB & Alpha-B crystallin \\
\hline & & CXCR4 & $\mathrm{C}-\mathrm{X}-\mathrm{C}$ motif chemokine receptor 4 \\
\hline \multirow{11}{*}{$\triangle$} & Alicia Forma & CXCR7 & C-X-C motif chemokine receptor 7 \\
\hline & aforma@onet.pl & $\mathrm{ECs}$ & Endothelial cells \\
\hline & Magdalena Tyczyńska & EGF & Epidermal growth factor \\
\hline & m.tyczynska@onet.pl & EMT & Epithelial-mesenchymal transition \\
\hline & Paweł Kedzierawski & FGFs & Fibroblast growth factors \\
\hline & pawelkedzierawski1@gmail.com & Fox & Mammalian forkhead box \\
\hline & Klaudyna Gietka & FOXO1 & Forkhead box $\mathrm{O} 1$ \\
\hline & klaudynka.gietka@gmail.com & $\mathrm{GC}$ & Gastric cancer \\
\hline & Monika Sitarz & HIF-1 and HIF-2 & Hypoxia-inducible factor 1 and 2 \\
\hline & mksitarz@gmail.com & HMGB1 & High-mobility group box-1 \\
\hline & & iNOS & Nitric oxide synthase \\
\hline \multirow[t]{2}{*}{1} & Department of Forensic Medicine, Medical University & KLF8 & Krüppel-like factor 8 \\
\hline & of Lublin, 20-090 Lublin, Poland & miRs & MicroRNAs \\
\hline \multirow[t]{2}{*}{2} & Department of Human Anatomy, Medical University & mTOR & Mammalian target of rapamycin \\
\hline & of Lublin, 20-090 Lublin, Poland & NGS & Next-generation sequencing \\
\hline 3 & $\begin{array}{l}\text { Department of Conservative Dentistry with Endodontics, } \\
\text { Medical University of Lublin, 20-090 Lublin, Poland }\end{array}$ & & \\
\hline
\end{tabular}




$\begin{array}{ll}\text { PA system } & \begin{array}{l}\text { Urokinase plasminogen activating } \\ \text { system }\end{array} \\ \text { PAR-2 } & \text { Proteinase-activated receptor-2 } \\ \text { PDGF- } \beta & \text { Platelet-derived growth factor-B } \\ \text { PI3K } & \text { Phosphatidyl inositol-3-kinase } \\ \text { PIC- } \gamma & \text { Phospholipase C- } \gamma \\ \text { PIGF } & \text { Placental growth factor } \\ \text { PTX3 } & \text { Pentraxin 3 } \\ \text { RELM- } \alpha & \text { Resistin-like-molecule-alfa } \\ \text { RUNX3 } & \text { Runt-related transcription factor 3 } \\ \text { SDF-1 } & \text { Stromal cell-derived factor-1 } \\ \text { STAT-3 } & \text { Signal transducer and activator of } \\ & \text { transcription 3 } \\ \text { TAMs } & \text { Tumor-associated macrophages } \\ \text { TM } & \text { Thymidine phosphorylase } \\ \text { VCAM-1 } & \text { Vascular endothelial cell adhesion } \\ & \text { molecule-1 } \\ \text { VEGF } & \text { Vascular endothelial growth factor } \\ \text { VEGF-A } & \text { Vascular endothelial growth factor A } \\ \text { VEGFR2 } & \text { Vascular endothelial growth factor } \\ & \text { receptor 2 } \\ \text { WHO } & \text { World Health Organization }\end{array}$

\section{Introduction}

Gastric cancer (GC) is the fourth most prevalent cancer worldwide and the second most often cause of cancerrelated deaths with more than 990,000 new diagnoses and approximately 738,000 death cases reported annually $[1,2]$. Males are reported to be nearly two to three times more susceptible to the onset of GC compared to females [3, 4]. The epidemiological studies showed that over $50 \%$ of the newly diagnosed patients are from developing countries such as Eastern Europe, East Asia, as well as Central and South America; lower risk areas include Southern Asia, East Africa, and North America [5]. When it comes to the 5-year survival rate, only Japan achieved mildly good results, whereas the European ratio oscillates between 10 and 30\% [6]. Throughout the last few decades, it has been observed that GC incidence rates decreased in most parts of the world; however, this data subject only to the sporadic intestinal type of GC; the prevalence of diffuse GC has increased [7]. Currently, more cases of the proximal GC than the distal one are observed among patients [8]. Numerous risk factors contribute to the onset of GC, among which Helicobacter pylori (H. pylori) infection, genetic factors, imbalanced diet, as well as excessive alcohol consumption, are of the highest importance [9-14].

Currently, two major histological classifications of GC are distinguished - the most commonly used - the Lauren classification and the most detailed one- the World Health Organization (WHO) classification [15]. Besides, another division that concerns the patient's age was proposed-an early-onset gastric carcinoma is mostly identified in patients aged 45 or younger, whereas, the conventional GC-in patients older than $45[16,17]$. In addition, another classification based on the next-generation sequencing (NGS) was proposed and it includes four major molecular subtypesMSI, MSS/TP53 ${ }^{+}$, MSS/TP53-, and MSS/EMT [18].

Gastric carcinogenesis is a complex, multifactorial process that is primarily stimulated by chronic inflammation (induced by $H$. pylori infection in the majority of cases); however, other processes such as the epithelial-mesenchymal transition (EMT) or intensified angiogenesis play a crucial role in GC progression [19-23]. Angiogenesis is a physiological process of the formation of the new blood vessels from the already existing ones. Apart from its relevance under the physiological conditions, angiogenesis is also crucial for the growth of the cancerous tissues, as the newly formed vessels provide nutrition, growth factors, and oxygen supply [24, 25]. Under physiological conditions, angiogenesis is mainly a consequence of the hypoxic and ischemic signals, whereas, in the pathological conditions, angiogenesis is uncontrolled and upregulated due to the predominance of the pro-angiogenic factors [26, 27]. Pathological angiogenesis is characterized by the atypical morphology of the tumor vasculature, as well as the increased proliferation of the endothelial cells (ECs), pericytes, smooth muscle cells, and the basement membrane [28]. Tumor-associated vessels differ from the normal vessels both morphologically and functionally. Physiological vessels are usually evenly distributed and due to their effective coating by the pericytes, they are able to provide efficient delivery of the nutrients and oxygen. Contrarily, tumor vessels are often inefficient because of the structural transformations-they tend to be unevenly distributed and form tortuous and overall chaotic networks with an irregular branching. Moreover, tumor vasculature is not perfused regularly and it presents a bidirectional blood flow [29, 30]. The progress and development of the cancerous tissues and their metastases result from an efficient vascular response. The process starts with an "angiogenic switch" that enables tumor growth and expansion beyond the primary site of tumorigenesis. The switch consists of the following steps: perivascular detachment and vessel dilation, angiogenic sprouting, creation of the new blood vessels, as well as the recruitment of the perivascular cells [31, 32]. Those processes can occur during every stage of the carcinogenesis-before, during, or even after tumor progression [33, 34].

Treatment strategies of GC include surgery, radiation, therapy, chemoradiation, chemotherapy, and targeted therapy. Some research demonstrated that metformin might also constitute a potential treatment for several types of cancer including GC [35-37]. Other potential strategies for the advanced GC include the decrease of the pro-angiogenic 
ligands levels and the expression of their receptors, the increase of the angiogenic inhibitors levels, as well as directly targeting the inner walls of the ECs [38]. Furthermore, the inhibition of the angiogenesis indirectly enhances the effects of chemotherapy, most likely because of the vascular normalization and more effective delivery of the chemotherapeutic agents (Table 1) [39-41]. The purpose of this review is to accentuate and evaluate the significance of the pro-angiogenic pathways in GC patients. Furthermore, we aimed to summarize the most meaningful pro-angiogenic factors and their receptors, as well as potential molecular pathways and their involvement in gastric carcinogenesis.

\section{Biomarkers of angiogenesis}

The carcinogenesis is associated with the release of the specific biomarkers that can be used for diagnosis, prognosis, and even selection of the proper treatment therapy. In GC, tumor cells release large amounts of the molecules that induce the growth of the new blood vessels, promoting the angiogenesis process. Studies have shown that GC cells present a high angiogenic potential by secreting the proangiogenic cytokines (such as angiopoietin-1, -2, -3, -4, and tryptase) that stimulate the ECs and the stromal cells, as well as the autocrine loop [42]. Specific biomarkers of angiogenesis might be crucial in assessing a patient's response to the particular anti-angiogenic therapy and predicting the overall clinical outcome of patients. Currently, the most described and best-known biomarkers of angiogenesis include the vascular endothelial growth factor and epidermal growth factor families, placental growth factor (PIGF), resistinlike-molecule- $\alpha$ (RELM- $\alpha$ ), angiopoietins, platelet-derived growth factor- $\beta$ (PDGF- $\beta$ ), fibroblast growth factor (FGF), hypoxia-inducible factor (HIF), tryptase, integrins, and IL-8.

\section{Vascular endothelial growth factor family}

Vascular endothelial growth factors (VEGFs) constitute a group of polypeptides that are considered to be one of the most crucial factors involved in the angiogenesis processes, which expression is significantly increased during gastric carcinogenesis [43]. Some of the members of the VEGF family are not only the pro-angiogenic factors crucial in the pathogenesis of the angiogenesis-related diseases, but play a role in the formation of the lymphatic vessels as well [44]. The VEGF family consists of the 7 major subtypes including VEGF-A, VEGF-B, VEGFC, VEGF-D, VEGF-E, VEGF-F, and PIGF [45]. VEGF family signaling is mediated by binding to the tyrosine kinase receptor (VEGFR-1, -2,-3), wherein VEGFR-2 is considered to be the main angiogenesis mediator. Vascular endothelial growth factor A (VEGF-A) is the first described cytokine that is considered to be the primary survival factor of the vascular ECs; VEGF-A stimulates their proliferation and migration, modulates the permeability, and inhibits the apoptosis [24, 46, 47]. VEGF-A, which is released in the excessive amounts from the tumour cells, is now considered as the strongest pro-angiogenic factor in gastric tumorigenesis $[48,49]$. The expression of VEGF-A

Table 1 The anti-angiogenic drugs that might be used in GC patients

\begin{tabular}{|c|c|c|c|}
\hline Anti-angiogenic drug & Biological category/mechanism of action & $\begin{array}{l}\text { Current } \\
\text { development } \\
\text { status* }\end{array}$ & Clinical indications \\
\hline Bevacizumab & Ani-VEGF human monoclonal antibody (IgG1) & Phase IV & $\begin{array}{l}\text { Gastric cancer, colorectal cancer, non-small cell } \\
\text { lung carcinoma, breast cancer, renal cell cancer, } \\
\text { glioblastoma multiforme, diabetic retinopathy, } \\
\text { macular degeneration, ovarian cancer }\end{array}$ \\
\hline Ramucirumab & $\begin{array}{l}\text { Anti-VEGFR-2 human monoclonal antibody } \\
\text { (IgG1) }\end{array}$ & Phase IV & $\begin{array}{l}\text { Gastric cancer, gastro-esophageal junction adeno- } \\
\text { carcinoma, colorectal cancer }\end{array}$ \\
\hline Sunitinib & Tyrosine kinase inhibitor & Phase II & $\begin{array}{l}\text { Gastric cancer, gastrointestinal stromal cancer, } \\
\text { meningioma, renal cell carcinoma, breast cancer, } \\
\text { non-small cell lung carcinoma, neuroendocrine } \\
\text { tumors, leukemia }\end{array}$ \\
\hline Sorafenib & Tyrosine kinase inhibitor & Phase II & $\begin{array}{l}\text { Gastric cancer, renal cell carcinoma, hepatocellular } \\
\text { carcinoma, thyroid cancer, desmoid tumors }\end{array}$ \\
\hline Apatinib & Tyrosine kinase inhibitor & Phase IV & $\begin{array}{l}\text { Gastric cancer, breast cancer, hepatocellular carci- } \\
\text { noma }\end{array}$ \\
\hline Aflibercept & VEGF-Trap; targets VEGF-A, VEGF-B, and PIGF & Phase II & $\begin{array}{l}\text { Gastric cancer, macular degeneration, colorectal } \\
\text { cancer }\end{array}$ \\
\hline Cetuximab & EGFR inhibitor & Phase III & Gastric cancer, squamous cell carcinoma \\
\hline
\end{tabular}

*For GC patients 
might increase the density and number of vessels in the intestinal as well as the diffuse-type of GC [50]. VEGF-A regulates vascular permeability and gene expression by the attachment and activation of the VEGFR-1 and VEGFR-2 [51]. Other VEGFs are also involved in the regulation of the angiogenesis and might be expressed either during embryogenesis or during adult stages usually under stressed or pathological conditions. Besides, Maeda et al. showed that patients with VEGF-positive tumours had a poorer prognosis compared to patients with VEGF-negative tumours [52].

\section{Vascular endothelial growth factor receptors}

VEGF receptor 1 (VEGFR1), VEGF receptor 2 (VEGFR2), and VEGF receptor 3 (VEGFR3) are tyrosine kinase receptors that constitute a site of attachment for the members of the VEGF family. These receptors are highly expressed on the ECs and allow VEGF family proteins to be released into the serum [43]. Except for angiogenesis, VEGFRs are also crucial in the infiltration of the immune cells into the tumor microenvironment, promoting persistent inflammation [53]. The binding of VEGFs to their specific receptors promotes angiogenesis by either the production of the signalling intermediates such as phosphatidyl inositol-3-kinase (PI3K), AKT, phospholipase C- $\gamma$ (PIC- $\gamma$ ), and small GTPases or by the induction of the pro-angiogenic signaling pathways including the PLC $\gamma$ PKC-MAPK pathway initiated by the VEGFR-2 [54, 55]. VEGF/VEGFR interactions also stimulate the mitogenesis and cellular migration as well as the recruitment and proliferation of the ECs.

\section{Epidermal growth factor family}

Epidermal growth factor (EGF) is a ligand that binds to the tyrosine kinase receptor (EGFR); its overexpression in solid tumours is associated with an enhanced progression of carcinogenesis and a poorer clinical outcome of patients. $\mathrm{EGF} / \mathrm{EGFR}$ is involved in the pro-angiogenic processes by regulating growth and maturation of the pathological vessels, stimulating the levels of VEGFR mRNA, as well as the enhanced neuropilin-1 (a coreceptor of VEGFR-2) release [54]. EGF activity is associated with the increased proliferation of the ECs, angiogenesis, as well as reduced apoptosis. The pro-angiogenic activity of EGF is facilitated due to the induction of several pro-oncogenic signalling pathways such as the RAS-RAF-MEK-ERK MAPK and AKT-PI3K-mTOR pathways [56, 57]. Except for angiogenesis, some of the signalling pathways such as TGF $\alpha$-EGFR, stimulate the formation of the lymphatic vessels and metastasis [55].

\section{Placental growth factor}

Placental grow factor (PIGF), a member of the VEGF family, controls the trophoblast growth and differentiation, and it has recently gained interest in carcinogenesis, because of its possible involvement in the angiogenic processes of several solid tumors and leukemia [58-61]. PIGF is mainly overexpressed during embryogenesis and stimulates angiogenesis and vasculogenesis; however, its overexpression during adulthood might induce pathological pro-angiogenic processes during carcinogenesis. PIGF presents a high affinity to VEGFR-1. Researchers demonstrated that among patients with GC, PIGF expression is highly associated with the metastases to lymph nodes, tumour stages, and poor overall survival $[61,62]$. The upregulation of the PIGF levels affects the VEGF/VEGFR pathway that further stimulates the proangiogenic pathways, the proliferation of ECs, as well as the enhanced inflammation.

\section{Resistin-like molecule-a}

Resistin-like molecule- $\alpha$ (RELM- $\alpha$ ) is a marker of the antiinflammatory macrophages and is highly related to the GC progression. Chen et al. showed that RELM- $\alpha$ expression is associated with the size of the tumour and its advanced stage [63]. The researchers also found that RELM- $\alpha$ activates VEGF by activating the NF- $\kappa B-M M P-9 / V E G F$ pathway that facilitates angiogenesis. Besides, RELM- $\alpha$ increases the expression of the other pro-angiogenic factors such as VEGF or vascular endothelial cell adhesion molecule-1 (VCAM-1) [64]. RELM- $\alpha$ is now considered as a novel biomarker of GC which inhibition might potentially reduce the progression and invasiveness of GC.

\section{Angiopoietins}

Angiopoietins (Angs) constitute a family of the angiogenic biomarkers of GC that belong to the family of vascular growth factors and are involved in the control of the embryonic as well as postnatal angiogenesis processes. Angs (Ang$1,-2,-3,-4)$ bind to the tyrosine kinase receptor tie-2 and are crucial for vessel maturation, migration, adhesion, and the survival of ECs. Ang-2 which disrupts the connections between the perivascular cells and the endothelium promotes cell death with vascular regression and that results in its critical role in tumor angiogenesis [65-67]. Angiopoietin-1 (Ang-1) and angiopoietin-2 (Ang-2) are currently the most examined and are known to be firmly expressed in GC [43, 68]. Both Angs are ligands for Tie-2. By activating Tie2, Ang-1 stabilizes the vessels by recruiting pericytes [69]. Contrarily, Ang-2 antagonizes Tie-2, thus prevents Ang-1 maturation and has an impact on the growth and maturation of the vessels [70, 71]. Imbalances between Ang-1 and 
Ang-2 levels might occur independently of each other which is further associated with the severity of the pro-angiogenic processes. The angiopoietin/Tie-cascade is one of the signalling pathways that is involved in the regulation of tumor angiogenesis.

\section{Platelet-derived growth factor- $\beta$}

Platelet-derived growth factor- $\beta$ (PDGF- $\beta$ ) belongs to the PDGF family that binds to the PDGFR- $\beta$ homodimer. PDGF- $\beta$ plays an important role in the progression of tumours as they can induce both-the angiogenesis and EMT-that are crucial in tumorigenesis [72-74]. Moreover, Suzuki et al. found that PDGF- $\beta$ and VEGF have been secreted simultaneously in gastric tumours; however, PDGF- $\beta$ was more crucial than VEGF in the maintenance of the vessels in the intestinal type of GC [75]. PDGF- $\beta$ is highly expressed by the ECs and also promotes the recruitment of the perivascular cells. Besides, PDGF- $\beta$ plays a role in the migration, proliferation, and adhesion of the endothelial progenitor cells, as well as the increased VEGF expression within the tumor microenvironment, that are crucial for both neovascularization and re-endothelialization [76].

\section{Fibroblast growth factors}

Fibroblast growth factors (FGFs) constitute a family of ligands that stimulate and regulate neovascularization and revascularization. FGF binds to four different receptors (FGFR -1, -2, -3, and -4) and regulates cell proliferation, migration, and survival. FGF-1 and FGF-2, by the activation of the AKT pathway, are considered to be one of the most important angiogenesis controllers [77, 78]. There is an association between the activity of FGFs, VEGFs, and inflammatory cytokines, and this synergy significantly facilitates the vascularization, enhancing tumor growth and progression [79]. FGFs facilitate the proliferation of the hypoxic ECs, initiating inflammation; besides, FGFs initiate the 'angiogenic phenotype' of the ECs and stimulate them to release a urokinase-type plasminogen activator with a proangiogenic activity [80]. Moreover, FGFs are reported to be the potential survival factors of the vascular cells [81]. It was demonstrated that the interaction between FGFs and pentraxin 3 (PTX3) might be related to angiogenesis during the growth of the cancerous tissues [82].

\section{Hypoxia-inducible factors}

Since the hypoxic conditions act as a main driving force for the angiogenesis, the pro-angiogenic activity within the tumor microenvironment is also regulated by the hypoxiainducible factors 1 and 2 (HIF-1 and HIF-2). The two isoforms of HIF-HIF- $1 \alpha$ and HIF- $2 \alpha$ - are mainly involved in the pro-angiogenic processes during tumorigenesis. The major role of HIF-1 is to monitor the cellular response to the oxygen levels in solid tumors, whereas HIF-2 is primarily involved in the regulation of the erythropoietin levels [83, 84]. HIF induces the expression of numerous pro-angiogenic factors including VEGFs, PDGF- $\beta$, plasminogen activator-inhibitor-1(PAI-1), and Angs (1 and 2), as well as regulates the remodeling of the pathophysiological vessels [85]. Besides, the overexpression of HIF-1 $\alpha$ is associated with the mutations of the several oncogenes (p54, PTEN, or VHL) and thus, HIF- $1 \alpha$ overexpression significantly affects the tumour growth, metastasis, and invasion properties [86]. HIF stimulates physiological neovascularization under the hypoxic conditions; however, its overexpression leads to the pathological pro-angiogenic processes, which for example is observed as excessive recruitment, stimulation, and proliferation of ECs, or the regulation of the pro-angiogenic genes expression [87].

\section{Tryptase}

Apart from the above-mentioned and well-known pro-angiogenic activators, recent research showed that tryptase, a nonclassical moderator, can stimulate angiogenesis both in vitro and in vivo. Tryptase stimulates ECs proliferation and activation of the proteinase-activated receptor-2 (PAR-2) with VEGF as a final product of this process [88, 89]. Tryptase is also involved in the overactivation of the endothelial progenitor cells via AKT and ERK signalling pathways [90]. Moreover, tryptase presents an ability to alter the composition of the extracellular matrix which facilitates angiogenesis [91]. The presence of the tryptase-positive mast cells within the tumor microenvironment is crucial in the initiation of the pathological angiogenesis [92, 93]. Tryptase-positive mast cells activate the c-Kit receptor pathway that further stimulates the gastric carcinogenesis [94]. Thus, tryptase affects angiogenesis either directly or indirectly and might constitute a potential biomarker of the angiogenesis in several types of cancers including GC.

\section{Integrins}

Other molecules_-integrins - significantly affect the interactions between the tumor and stromal tissues [95]. The integrins involved in the tumor angiogenesis include several heterodimers such as $\alpha 1 \beta 1, \alpha 2 \beta 1, \alpha 4 \beta 1, \alpha 5 \beta 1, \alpha 6 \beta 1, \alpha 6 \beta 4$, $\alpha 9 \beta 1, \alpha v \beta 3$, and $\alpha v \beta 5$. Integrins play a pivotal role in the regulation of the ECs migration and survival, as well as the enhanced recruitment of the monocytes to the tumor microenvironment [96]. Tumor angiogenesis is also facilitated by the stimulation of the VEGF pathways by integrins and the integrins-related reorganization of the extracellular matrix promoting the microenvironment that is more prone to the 
pro-angiogenic alterations. Except for angiogenesis, integrins are also involved in the regulation of the lymphangiogenesis and both of these processes significantly affect the tumor metastasis to other organs, worsening the clinical outcome of patients [97]. Therefore, it was proposed that integrins might act as potential biomarkers of angiogenesis and their inhibition alone or combined with other treatment strategies might be crucial during GC therapy.

\section{Interleukin-8}

Interleukin-8 (IL-8) is one of the pro-angiogenic chemokines released by the tumor-infiltrating macrophages involved in the regulation of angiogenesis in various types of cancers. The pro-angiogenic effects of the IL-8 action are stimulated by both-the paracrine and autocrine routes in carcinogenesis. IL-8 induces the overexpression of the VEGF-A, VEGFR-1, and VEGFR-2 suggesting that it might act as a potential biomarker of angiogenesis in GC patients [98]. IL-8 also stimulates the proliferation, survival, and migration of the ECs via the Src/Vav2/Rac1/PAK1 signaling pathway [99]. Besides, IL-8 is involved in the regulation of the capillary tube organization [100]. IL-8 is believed to be a promising marker of several gastrointestinal cancers including $\mathrm{GC}$ or colorectal cancer.

\section{Angiogenic pathways in gastric cancer}

Angiogenesis is a complex process that is continually stimulated by the numerous pro-angiogenic and anti-angiogenic factors, as well as molecular pathways enabling further progression of gastric carcinogenesis, growth of the cancerous tissues, and metastasis (Fig. 1). So far, a great number of the pro-angiogenic factors that contribute to gastric carcinogenesis have been described. VEGFR-2, activated by the VEGF attachment, facilitates the formation of the new blood vessels providing oxygen supply and essential nutrients for the gastric cancerous tissues. Moreover, VEGFR-2 also regulates survival, progression, and invasion of GC in a VEGF-independent manner [101]. It was demonstrated that the interaction between stromal cell-derived factor-1 (SDF-1) and the C-X-C motif chemokine receptor (CXCR7) induces the secretion of the significant amounts of VEGF

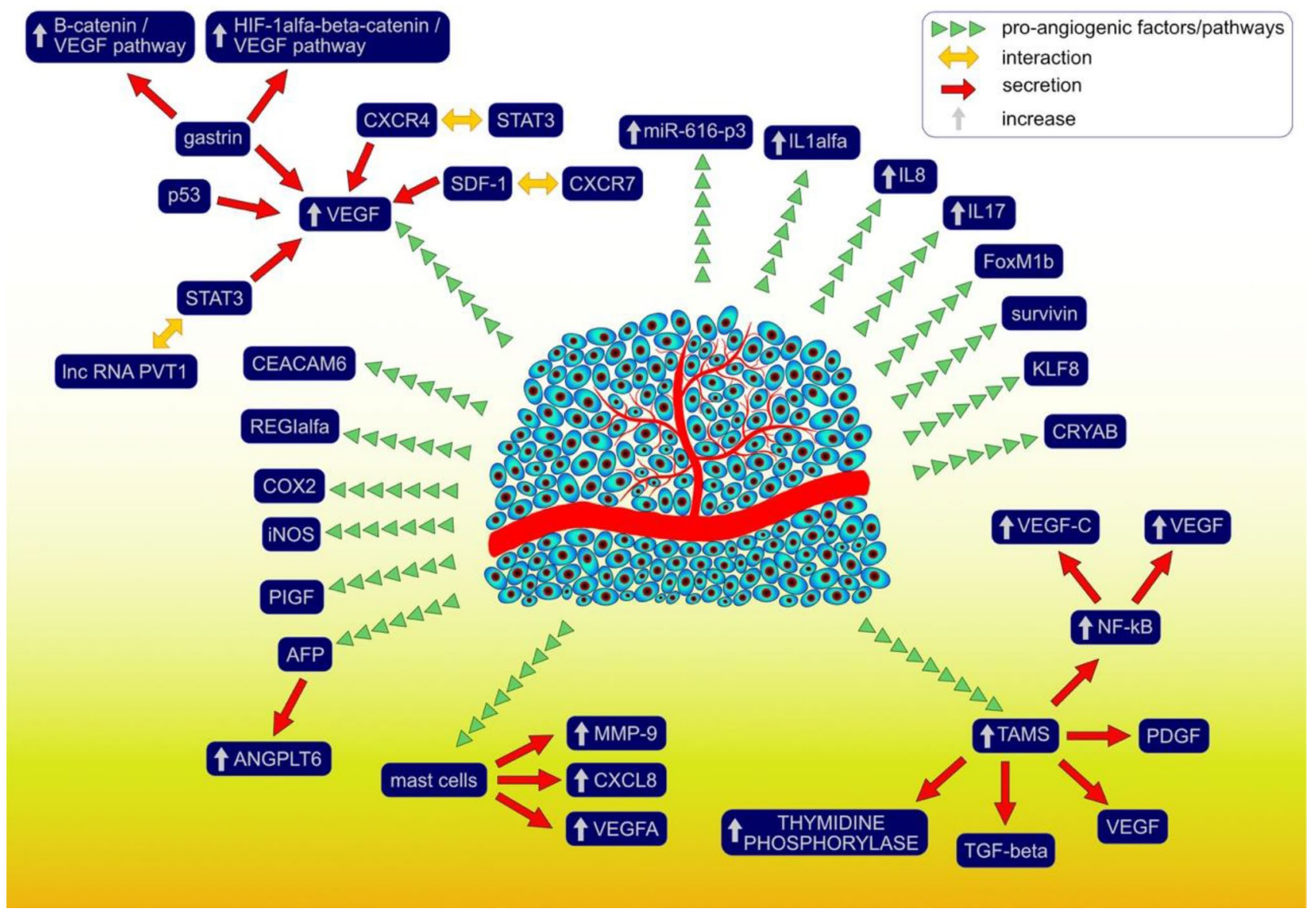

Fig. 1 Molecular factors and pathways that are involved in the angiogenesis process during gastric carcinogenesis 
[102]. Besides, the knockdown of CXCR7 prevents further VEGF release and thus, it might constitute one of the potential molecular targets preventing the progression of angiogenesis. Except for angiogenesis, the SDF-1/CXCR7 pathway is also involved in the proliferation, adhesion, and invasion of the tumor cells. Another C-X-C motif chemokine receptor-CXCR4-promotes gastric carcinogenesis via a signal transducer and the activator of transcription 3 (STAT3)-dependent upregulation of the VEGF levels [103]. Gastrin enhances angiogenesis in either normoxic or hypoxic conditions eventually upregulating the VEGF levels; under normoxic conditions, gastrin promotes the activation of the $\beta$-catenin/VEGF pathway, whereas, under the hypoxic conditions, HIF- $1 \alpha / \beta$-catenin/VEGF pathway is overactivated [104]. HIF- $1 \alpha$ regulates the expression of the pro-angiogenic genes such as $V E G F$, significantly contributing to the regulation of the angiogenesis process. VEGFA expression is also upregulated by the interaction between IncRNA PVT1 and the STAT3 pathway; except for the pro-angiogenic effects, the oncogenic effects are further enhanced by the continuous stimulation of PV1 transcription by the activated STAT3 pathway [105]. Besides, aberrant expression of one of the tumor suppressor genes- $\mathrm{p} 53$, might promote angiogenesis via the upregulation of the VEGF levels at the same time decreasing the amounts of thrombospondin- 1 that acts as angiogenesis inhibitor [106]. The abovementioned finding is of high importance during tumorigenesis since the majority of cancers are characterized by either the loss of or dysregulated p53 functions; $\mathrm{p} 53$ is the most frequently mutated gene. VEGF expression can also be increased by the urokinase plasminogen activating system (PA system) contributing to the pro-angiogenic activities and further tumor progression and invasion [107].

Tumor-associated macrophages (TAMs) that have an M2-like phenotype have an ability to induce angiogenesis and promote the proliferation of the cancer cells within the gastric microenvironment. Except for angiogenesis, TAMs also stimulate lymphangiogenesis and those two processes might occur simultaneously. TAMs induce the release of numerous pro-angiogenic factors including TGF- $\beta$, VEGF, PDGF, as well as angiogenic chemokines [108, 109]. TAMs promote NF-kB activation and further overexpression of the VEGF and VEGF-C levels promoting angiogenesis and lymphangiogenesis in the GC tissues [110]. Moreover, there is a correlation between patients' serum VEGF and VEGF-C levels and the number of TAMs observed within the cancerous specimens. Park et al. (2015) showed that CD163 + TAMs promote angiogenesis and induce greater CXCL12 expression in GC patients [111]. The overexpression of CXCL12 stimulates further recruitment of TAMs into the gastric microenvironment inducing tumor cell invasion and progression of gastric carcinogenesis. Besides, TAMs that are characterized by the overexpression of the thymidine phosphorylase (TM) (an angiogenesis-promoting enzyme) levels, are associated with greater tumor angiogenesis and worse clinical outcome and survival of patients with the intestinal type of GC [112]. Other cells that contribute to the progression of angiogenesis include mast cells releasing great amounts of the pro-angiogenic factors such as VEGFA, CXCL8, or MMP-9 [113]. Except for angiogenesis, mast cells are also involved in the lymphangiogenesis during GC progression. There is a correlation between the extent of angiogenesis as well as malignancy grade and the amounts of the mast cells infiltrating the cancerous tissues. Ribatti et al. (2010) showed that the density of the tryptase-positive mast cells was significantly greater than chymase-positive mast cells in the examined GC specimens; however, it should be considered that all of the mast cells express tryptase activity [114]. Tryptase, as MMP-9, degrades gelatin, thus, facilitates the angiogenesis. Nevertheless, the greater cumulation of the chymase-positive cells can also promote angiogenesis providing further tumor growth primarily by the MMP-9 activation [115].

The expression of cyclooxygenase-2 (COX-2) that is usually highly abundant in GC tissues correlates with the enhanced angiogenesis also contributing to the greater proliferation of GC cells and lymph node metastasis [116]. COX-2 expression also promotes the apoptosis of the GC cells affecting the overall outcome [117]. COX-2 stimulates angiogenesis primarily by modulating the release of VEGF and basic fibroblast growth factor [118]. It was shown that COX-2 inhibitors might significantly reduce angiogenesis, at the same time suppressing the proliferation of the cancer cells and weakening the invasiveness $[119,120]$. COX-2 as well as nitric oxide synthase (iNOS), except for stimulating neovascularization and cancer progression, can also act as potential biomarkers indicating the survival of GC patients [121, 122].

Regulation and progression of angiogenesis are also controlled by the regenerating gene (REG) I $\alpha$ that also stimulates the growth of the cancerous tissues and the antiapoptotic effects on the endothelial cells [123]. GCs that produce high amounts of $\alpha$-fetoprotein (AFP) are more prone to induce rich neovascularization, high proliferative activity, and weak apoptosis compared to AFP-negative GCs [124]. AFP-positive cancers are considered to be associated with poorer clinical outcome and prognosis of GC patients. Besides, AFP-positive cancers express high levels of ANGPTL6 that additionally promotes tube formation and ECs migration via the activation of the ERK $1 / 2$ and AKT pathways [125]. GC metastasis and angiogenesis can also be enhanced by the overexpression of the carcinoembryonic antigen-related cell adhesion molecule 6 (CEACAM6) and further induction of the FAK signaling pathway [126]. The expression of the PIGF, a member of the VEGF family, is also associated with greater vascularization, lymph 
node metastasis, serosal invasion, and the general clinical outcome of patients [127]. Other factors that might promote gastric angiogenesis either directly or indirectly include survivin, the mammalian Forkhead Box (Fox) transcription factor FoxM1b, Krüppel-like factor 8 (KLF8), or alpha-B crystallin (CRYAB) [128-131]. The inactivation of the PTEN gene might also contribute to the enhanced vascularization process [132]. The regulation of several proangiogenic factors such as VE-cadherin, MMPs-2 and 9, MT1-MMP, p-ERK, $\beta$-catenin, 23 p-FAK, or p-paxillin was reported to be controlled by an oncogenic long non-coding RNA-MALAT1-which overexpression correlates with poor clinical outcome of GC patients [133]. Angiogenesis is also regulated and stimulated by a vast number of interleukins and their receptors among which IL-1 $\alpha$, IL-8 (via high-mobility group box-1 (HMGB1) contribution), IL-17 (through STAT3 pathway), or soluble interleukin-2 receptor (sIL-2R) are considered to be of the highest importance regarding GC patients; except for neovascularization, IL- $1 \alpha$ is involved in liver metastasis of GC [134-137].

The dysregulation of microRNAs (miRs) has also been demonstrated to play a significant role in the induction and progression of angiogenesis in GC patients. GC tissues express the upregulated levels of miR-616-3p that significantly contribute to the induction and progression of angiogenesis as well as the EMT process primarily via the activation of the PTEN/AKT/mTOR pathway [138]. MiR-130a and miR-495 downregulate the Runt-related transcription factor 3 (RUNX3) expression ultimately enhancing angiogenesis and gastric cell proliferation [139]. VEGF-A is primarily targeted by miR-125a and miR-126, facilitating vascularization; other mechanisms of miR-related angiogenesis include the forkhead box O1 (FOXO1) inhibition by miR-135b [140-142]. Contrarily, enhanced expression of miR-218 contributes to the inhibition of the tumor growth by preventing vascularization via targeting ROBO1 [143].

\section{Conclusions}

Angiogenesis constitutes a crucial component of a complex gastric carcinogenesis process enabling quicker and more effective growth of the cancerous tissues. The understanding of the mechanisms that drive angiogenesis during gastric carcinogenesis constitutes a basic approach to provide potential anti-angiogenic therapies. The majority of the currently available anti-angiogenic therapies for GC patients include those that primarily focus on the VEGF-VEGFR pathway specifically. Besides, the pro-angiogenic factors described in this review differ in terms of their clinical significance and application; in GC patients, the expression of VEGFR-2, CXCR7, EGF, VEGF, IL-8, miRNAs (such as miR-218, miR-135b, miR-495, or miR-130a), or TAMs proliferation seem to be associated with the survival rates, thus, they might act as potential prognostic factors of GC. The molecular components and pathways described in this review provide an insight into a vast number of the proangiogenic mechanisms that should be further studied to improve the available treatment strategies. The factors and pathways described in this review might also constitute potential molecular targets for establishing new effective anti-angiogenic therapies for GC patients.

\section{Compliance with ethical standards}

Conflict of interest Alicja Forma, Magda Tyczyńska, Paweł Kędzierawski, Klaudyna Gietka, and Monika Sitarz declare that they have no conflict of interest.

Human and/or animal rights This study does not include any data about human subjects.

Informed consent This study does not involve human subjects and does not apply to giving Informed Consent.

Open Access This article is licensed under a Creative Commons Attribution 4.0 International License, which permits use, sharing, adaptation, distribution and reproduction in any medium or format, as long as you give appropriate credit to the original author(s) and the source, provide a link to the Creative Commons licence, and indicate if changes were made. The images or other third party material in this article are included in the article's Creative Commons licence, unless indicated otherwise in a credit line to the material. If material is not included in the article's Creative Commons licence and your intended use is not permitted by statutory regulation or exceeds the permitted use, you will need to obtain permission directly from the copyright holder. To view a copy of this licence, visit http://creativecommons.org/licenses/by/4.0/.

\section{References}

1. Ferlay J, Shin H-R, Bray F, et al. Estimates of worldwide burden of cancer in 2008: GLOBOCAN 2008. Int J Cancer. 2010;127:2893-917.

2. Edwards BK, Noone AM, Mariotto AB, et al. Annual Report to the Nation on the status of cancer, 1975-2010, featuring prevalence of comorbidity and impact on survival among persons with lung, colorectal, breast, or prostate cancer. Cancer. 2014;120:1290-314

3. Jemal A, Center MM, DeSantis C, et al. Global patterns of cancer incidence and mortality rates and trends. Cancer Epidemiol Biomarkers Prev. 2010;19:1893-907.

4. Sitarz R, Skierucha M, Mielko J, et al. Gastric cancer: epidemiology, prevention, classification, and treatment. Cancer Manag Res. 2018;10:239-48.

5. Machlowska J, Baj J, Sitarz M, et al. Gastric cancer: epidemiology, risk factors, classification, genomic characteristics and treatment strategies. Int J Mol Sci. 2020;21:4012.

6. Matsuda T, Saika K. The 5-year relative survival rate of stomach cancer in the USA, Europe and Japan. Jpn J Clin Oncol. 2013;43:1157-8. 
7. Ferro A, Peleteiro B, Malvezzi M, et al. Worldwide trends in gastric cancer mortality (1980-2011), with predictions to 2015, and incidence by subtype. Eur J Cancer. 2014;50:1330-44.

8. Fan X-S. Differences in HER2 over-expression between proximal and distal gastric cancers in the Chinese population. World $\mathbf{J}$ Gastroenterol. 2013;19:3316.

9. Guggenheim DE. Shah MA Gastric cancer epidemiology and risk factors. J Surg Oncol. 2012;107:230-6.

10. Pormohammad A, Ghotaslou R, Leylabadlo HE, et al. Risk of gastric cancer in association with Helicobacter pylori different virulence factors: a systematic review and meta-analysis. Microb Pathog. 2018;118:214-9.

11. Zali H, Rezaei-Tavirani M, Azodi M. Gastric cancer: prevention, risk factors and treatment. Gastroenterol Hepatol Bed Bench. 2011;4:175-85.

12. Grochowski C, Blicharska E, Baj J, et al. Serum iron, magnesium, copper, and manganese levels in alcoholism: a systematic review. Molecules. 2019;24:1361.

13. Baj J, Flieger W, Teresiński G, et al. Magnesium, calcium, potassium, sodium, phosphorus, selenium, zinc, and chromium levels in alcohol use disorder: a review. J Clin Med. 2020;29:1901.

14. Grochowski C, Blicharska E, Bogucki J, et al. Increased aluminum content in certain brain structures is correlated with higher silicon concentration in alcoholic use disorder. Molecules. 2019;24:1721.

15. Hu B, El Hajj N, Sittler S, et al. Gastric cancer: classification, histology and application of molecular pathology. J Gastrointest Oncol. 2012;3:251-61.

16. Milne AN, Sitarz R, Carvalho R, et al. Early onset gastric cancer: on the road to unraveling gastric carcinogenesis. Curr Mol Med. 2007;7:15-28.

17. Milne AN, Carvalho R, Morsink FM, et al. Early-onset gastric cancers have a different molecular expression profile than conventional gastric cancers. Mod Pathol. 2006;19:564-72.

18. Machlowska J, Kapusta P, Baj J, et al. High-throughput sequencing of gastric cancer patients: unravelling genetic predispositions towards an early-onset subtype. Cancers. 2020;12:1981.

19. Pucułek M, Machlowska J, Wierzbicki R, et al. Helicobacter pylori associated factors in the development of gastric cancer with special reference to the early-onset subtype. Oncotarget. 2018;9:31146-62.

20. Ciaula AD, Baj J, Garruti G, et al. Liver steatosis, gut-liver axis, microbiome and environmental factors. A never-ending bidirectional cross-talk. J Clin Med. 2020;9:2648.

21. Baj J, Brzozowska K, Forma A, et al. Immunological aspects of the tumor microenvironment and epithelial-mesenchymal transition in gastric carcinogenesis. Int J Mol Sci. 2020;21:2544.

22. Baj J, Korona-Głowniak I, Forma A, et al. Mechanisms of the epithelial-mesenchymal transition and tumor microenvironment in helicobacter pylori-induced gastric cancer. Cells. 2020;9:1055.

23. Pucułek M, Baj J, Portincasa P, et al. The morphology and application of stem cells in digestive system surgery. Folia Morphologica 2020.

24. Folkman J. Tumor angiogenesis: therapeutic implications. N Engl J Med. 1971;285:1182-6.

25. Saaristo A, Karpanen T, Alitalo K. Mechanisms of angiogenesis and their use in the inhibition of tumor growth and metastasis. Oncogene. 2000;19:6122-9.

26. Yadav L, Puri N, Rastogi V, et al. Tumour angiogenesis and angiogenic inhibitors: a review. J Clin Diagn Res. 2015;9:XE01-5.

27. Hoff PM, Machado KK. Role of angiogenesis in the pathogenesis of cancer. Cancer Treat Rev. 2012;38:825-33.

28. Loizzi V, Vecchio VD, Gargano G, et al. Biological pathways involved in tumor angiogenesis and bevacizumab based antiangiogenic therapy with special references to ovarian cancer. Int J Mol Sci. 2017;18:1967.
29. Ziyad S, Iruela-Arispe ML. Molecular mechanisms of tumor angiogenesis. Genes Cancer. 2011;2:1085-96.

30. Nagy JA, Chang S-H, Shih S-C, et al. Heterogeneity of the tumor vasculature. Semin Thromb Hemost. 2010;36:321-31.

31. Bergers G, Benjamin L. Tumorigenesis and the angiogenic switch. Nat Rev Cancer. 2003;3:401-10.

32. Hanahan D, Folkman J. Patterns and emerging mechanisms of the angiogenic switch during tumorigenesis. Cell. 1996;86:353-64.

33. Ilson DH. Angiogenesis in gastric cancer: hitting the target? Lancet. 2014;383:4-6.

34. Hsieh HL, Tsai MM. Tumor progression-dependent angiogenesis in gastric cancer and its potential application. World J Gastrointest Oncol. 2019;11:686-704.

35. Courtois S, Lehours $P$, Bessède $E$. The therapeutic potential of metformin in gastric cancer. Gastric Cancer. 2019;22:653-62.

36. Li X, Li T, Liu Z, et al. The effect of metformin on survival of patients with pancreatic cancer: a meta-analysis. Sci Rep. 2017;7:5825.

37. Mazurek M, Litak J, Kamieniak P, et al. Metformin as potential therapy for high-grade glioma. Cancers. 2020;12:210.

38. Carmeliet P, Jain RK. Molecular mechanisms and clinical applications of angiogenesis. Nature. 2011;473:298-307.

39. Ma J, Waxman DJ. Combination of antiangiogenesis with chemotherapy for more effective cancer treatment. Mol Cancer Ther. 2008;7:3670-84.

40. El-Kenawi AE, El-Remessy AB. Angiogenesis inhibitors in cancer therapy: mechanistic perspective on classification and treatment rationales. Br J Pharmacol. 2013;170:712-29.

41. Fuso Nerini I, Cesca M, Bizzaro F, et al. Combination therapy in cancer: effects of angiogenesis inhibitors on drug pharmacokinetics and pharmacodynamics. Chin J Cancer. 2016;35:61.

42. Nienhüser H, Schmidt T. Angiogenesis and anti-angiogenic therapy in gastric cancer. Int J Mol Sci. 2017;19:43.

43. Macedo F, Ladeira K, Longatto-Filho A, et al. Gastric cancer and angiogenesis: is VEGF a useful biomarker to assess progression and remission? J Gastric Cancer. 2017;17:1.

44. Holmes DI, Zachary I. The vascular endothelial growth factor (VEGF) family: angiogenic factors in health and disease. Genome Biol. 2005;6:209.

45. Leung DW, Cachianes G, Kuang WJ, et al. Vascular endothelial growth factor is a secreted angiogenic mitogen. Science. 1989;246:1306-9.

46. Zecchin A, Kalucka J, Dubois C, et al. How endothelial cells adapt their metabolism to form vessels in tumors. Front Immunol. 2017;8:1750.

47. Olsson AK, Dimberg A, Kreuger J, et al. VEGF receptor signalling-in control of vascular function. Nat Rev Mol Cell Biol. 2006;7:359-71.

48. Kitadai Y. Angiogenesis and lymphangiogenesis of gastric cancer. J Oncol. 2010;2010:468725.

49. Zhang X, Tang J, Zhi X, et al. miR-874 functions as a tumor suppressor by inhibiting angiogenesis through STAT3/VEGF-A pathway in gastric cancer. Oncotarget. 2015;6:1605-17.

50. Takahashi Y, Cleary KR, Mai M, et al. Significance of vessel count and vascular endothelial growth factor and its receptor (KDR) in intestinal-type gastric cancer. Clin Cancer Res. 1996;2:1679-84.

51. Shibuya M. Vascular endothelial growth factor (VEGF) and its receptor (VEGFR) signaling in angiogenesis: a crucial target for anti- and pro-angiogenic therapies. Genes Cancer. 2011;2:1097-105.

52. Maeda K, Chung Y-S, Ogawa Y, et al. Prognostic value of vascular endothelial growth factor expression in gastric carcinoma. Cancer. 1996;77:858-63.

53. Yang J, Yan J, Liu B. Targeting VEGF/VEGFR to modulate antitumor immunity. Front Immunol. 2018;9:978. 
54. Cruijsen HV, Giaccone G, Hoekman K. Epidermal growth factor receptor and angiogenesis: opportunities for combined anticancer strategies. Int J Cancer. 2005;117:883-8.

55. Sasaki T, Hiroki K, Yamashita Y. The role of epidermal growth factor receptor in cancer metastasis and microenvironment. Biomed Res Int. 2013;2013:1-8.

56. Wee $\mathrm{P}$, Wang Z. Epidermal growth factor receptor cell proliferation signaling pathways. Cancers (Basel). 2017;9:52.

57. Karar J, Maity A. PI3K/AKT/mTOR pathway in angiogenesis. Front Mol Neurosci. 2011;4:51.

58. Autiero M, Luttun A, Tjwa M, et al. Placental growth factor and its receptor, vascular endothelial growth factor receptor-1: novel targets for stimulation of ischemic tissue revascularization and inhibition of angiogenic and inflammatory disorders. J Thromb Haemost. 2003;1:1356-70.

59. Villarejo-Campos P, Padilla-Valverde D, Martin RM, et al. Serum VEGF and VEGF-C values before surgery and after postoperative treatment in gastric cancer. Clin Transl Oncol. 2013;15:265-70.

60. Fischer C, Mazzone M, Jonckx B, et al. FLT1 and its ligands VEGFB and PIGF: drug targets for anti-angiogenic therapy? Nat Rev Cancer. 2008;8:942-56.

61. Chen C-N, Hsieh F-J, Cheng Y-M, et al. The significance of placenta growth factor in angiogenesis and clinical outcome of human gastric cancer. Cancer Lett. 2004;213:73-82.

62. Aktaş SH, Akbulut $\mathrm{H}$, Yazici $\mathrm{O}$, et al. A new angiogenesis prognostic index with VEGFA, PIGF, and angiopoietin1 predicts survival in patients with advanced gastric cancer. Turk J Med Sci. 2017;47:399-406.

63. Chen P, Zhao D, Wang W, et al. High expression of RELM- $\alpha$ correlates with poor prognosis and promotes angiogenesis in gastric cancer. Oncol Rep. 2015;34:77-86.

64. Nair MG, Du Y, Perrigoue JG, et al. Alternatively activated macrophage-derived RELM- $\{$ alpha $\}$ is a negative regulator of type 2 inflammation in the lung. J Exp Med. 2009;206:937-52.

65. Schmidt T, Carmeliet P. Angiogenesis: a target in solid tumors, also in leukemia? Hematol Am Soc Hematol Educ Progr. 2011;2011:1-8.

66. Wang J, Wu KC, Zhang DX, et al. Antisense angiopoietin-1 inhibits tumorigenesis and angiogenesis of gastric cancer. World J Gastroenterol. 2006;12:2450-4.

67. Felcht M, Luck R, Schering A, et al. Angiopoietin-2 differentially regulates angiogenesis through TIE2 and integrin signaling. J Clin Invest. 2012;122:1991-2005.

68. Wang J, Wu K, Zhang D, et al. Expressions and clinical significances of angiopoietin-1, -2 and Tie2 in human gastric cancer. Biochem Biophys Res Commun. 2005;337:386-93.

69. Davis S, Aldrich TH, Jones PF, et al. Isolation of angiopoietin-1, a ligand for the TIE2 receptor, by secretion-trap expression cloning. Cell. 1996;87:1161-9.

70. Maisonpierre PC, Suri C, Jones PF, et al. Angiopoietin-2, a natural antagonist for Tie2 that disrupts in vivo angiogenesis. Science. 1997;277:55-60.

71. Sato TN, Tozawa Y, Deutsch U, et al. Distinct roles of the receptor tyrosine kinases Tie-1 and Tie-2 in blood vessel formation. Nature. 1995;376:70-4.

72. Guo Y, Yin J, Zha L, et al. Clinicopathological significance of platelet-derived growth factor B, platelet-derived growth factor recpertor- $\beta$, and E-cadherin expression in gastric carcinoma. Współczesna Onkol. 2013;2:150-5.

73. Christiansen JJ, Rajasekaran AK. Reassessing epithelial to mesenchymal transition as a prerequisite for carcinoma invasion and metastasis. Cancer Res. 2006;66:8319-26.

74. Folkman J. Role of angiogenesis in tumor growth and metastasis. Semin Oncol. 2002;29:15-8.
75. Suzuki S, Dobashi Y, Hatakeyama Y, et al. Clinicopathological significance of platelet-derived growth factor (PDGF)-B and vascular endothelial growth factor-A expression, PDGF receptor- $\beta$ phosphorylation, and microvessel density in gastric cancer. BMC Cancer. 2010;10:659.

76. Li H, Fredriksson L, Li X, et al. PDGF-D is a potent transforming and angiogenic growth factor. Oncogene. 2003;22:1501-10.

77. Fagiani E, Christofori G. Angiopoietins in angiogenesis. Cancer Lett. 2013;328:18-26.

78. Liang G, Liu Z, Wu J, et al. Anticancer molecules targeting fibroblast growth factor receptors. Trends Pharmacol Sci. 2012;33:531-41.

79. Presta M, Dell'Era P, Mitola S, et al. Fibroblast growth factor/fibroblast growth factor receptor system in angiogenesis. Cytokine Growth Factor Rev. 2005;16:159-78.

80. Montesano R, Vassalli JD, Baird A, et al. Basic fibroblast growth factor induces angiogenesis in vitro. Proc Natl Acad Sci U S A. 1986;83:7297-301.

81. Murakami M, Simons M. Fibroblast growth factor regulation of neovascularization. Curr Opin Hematol. 2008;15:215-20.

82. Presta M, Foglio E, Schuind AC, et al. Long pentraxin-3 modulates the angiogenic activity of fibroblast growth factor- 2 . Front Immunol. 2018;9:2327.

83. Forough R, Weylie B, Patel C, et al. Role of akt/pkb signaling in fibroblast growth factor-1 (fgf-1)-induced angiogenesis in the chicken chorioallantoic membrane (cam). J Cell Biochem. 2005;94:109-16.

84. Stoeltzing O, McCarty MF, Wey JS, et al. Role of hypoxiainducible factor 1alpha in gastric cancer cell growth, angiogenesis, and vessel maturation. J Natl Cancer Inst. 2004;96(12):946-56.

85. Hashimoto T, Shibasaki F. Hypoxia-inducible factor as an angiogenic master switch. Front Pediatr. 2015;3:33.

86. Shi YH, Fang WG. Hypoxia-inducible factor-1 in tumour angiogenesis. World J Gastroenterol. 2004;10:1082-7.

87. Krock BL, Skuli N, Simon MC. Hypoxia-induced angiogenesis: good and evil. Genes Cancer. 2011;2:1117-33.

88. Harris AL. Hypoxia-A key regulatory factor in tumour growth. Nat Rev Cancer. 2002;2:38-47.

89. Morris DR, Ding Y, Ricks TK, et al. Protease-activated receptor-2 is essential for factor via and xa-induced signaling, migration, and invasion of breast cancer cells. Cancer Res. 2006;66:307-14.

90. Qian N, Li X, Wang X, et al. Tryptase promotes breast cancer angiogenesis through PAR-2 mediated endothelial progenitor cell activation. Oncol Lett. 2018;16:1513-20.

91. de Souza Junior DA, Santana AC, da Silva EZ, et al. The role of mast cell specific chymases and tryptases in tumor angiogenesis. Biomed Res Int. 2015;2015:142359.

92. Guo X, Zhai L, Xue R, et al. Mast cell tryptase contributes to pancreatic cancer growth through promoting angiogenesis via activation of angiopoietin-1. Int J Mol Sci. 2016;17:834.

93. Ribatti D, Guidolin D, Marzullo A, et al. Mast cells and angiogenesis in gastric carcinoma. Int J Exp Pathol. 2010;91:350-6.

94. Ammendola M, Sacco R, Sammarco G, et al. Mast cells positive to tryptase and c-Kit receptor expressing cells correlates with angiogenesis in gastric cancer patients surgically treated. Gastroenterol Res Pract. 2013;2013:703163.

95. Ammendola M, Sacco R, Zuccala V, et al. Mast cells density positive to tryptase correlate with microvascular density in both primary gastric cancer tissue and loco-regional lymph node metastases from patients that have undergone radical surgery. Int J Mol Sci. 2016;17:905. 
96. Avraamides CJ, Garmy-Susini B, Varner JA. Integrins in angiogenesis and lymphangiogenesis. Nat Rev Cancer. 2008;8:604-17.

97. Chen $\mathrm{C}$, Chang $\mathrm{C}$, Lai $\mathrm{H}$, et al. Connective tissue growth factor inhibits gastric cancer peritoneal metastasis by blocking integrin $\alpha 3 \beta 1$-dependent adhesion. Gastric Cancer. 2015;18:504-15.

98. Shi J, Wei PK. Interleukin-8: a potent promoter of angiogenesis in gastric cancer. Oncol Lett. 2016;11:1043-50.

99. Ju L, Zhou Z, Jiang B, et al. Autocrine VEGF and IL-8 promote migration via Src/Vav2/Rac1/PAK1 signaling in human umbilical vein endothelial cells. Cell Physiol Biochem. 2017;41:1346-59.

100. Li A, Dubey S, Varney ML, et al. IL-8 directly enhanced endothelial cell survival, proliferation, and matrix metalloproteinases production and regulated angiogenesis. J Immunol. 2003; 170:3369-76.

101. Lian L, Li X-L, Xu M-D, et al. VEGFR2 promotes tumorigenesis and metastasis in a proangiogenic- independent way in gastric cancer. BMC Cancer. 2019;19:183.

102. Ma D-M, Luo D-X, Zhang J. SDF-1/CXCR7 axis regulates the proliferation, invasion, adhesion, and angiogenesis of gastric cancer cells. World J Surg Oncol. 2016;14:256.

103. Zhang Q, Xu F, Shi Y, et al. C-X-C motif chemokine receptor 4 promotes tumor angiogenesis in gastric cancer via activation of JAK2/STAT3. Cell Biol Int. 2017;41:854-62.

104. Tang E, Wang Y, Liu T, et al. Gastrin promotes angiogenesis by activating HIF- $1 \alpha / \beta$-catenin/VEGF signaling in gastric cancer. Gene. 2019;704:42-8.

105. Zhao J, Du P, Cui P, et al. LncRNA PVT1 promotes angiogenesis via activating the STAT3/VEGFA axis in gastric cancer. Oncogene. 2018;37:4094-109.

106. Joo YE, Sohn YH, Joo SY, et al. The role of vascular endothelial growth factor VEGFand p53 status for angiogenesis in gastric cancer. Korean J Intern Med. 2002;17:211-9.

107. Kaneko T, Konno H, Baba M, et al. Urokinase-type plasminogen activator expression correlates with tumor angiogenesis and poor outcome in gastric cancer. Cancer Sci. 2003;94:43-9.

108. Bingle L, Lewis CE, Corke KP, et al. Macrophages promote angiogenesis in human breast tumour spheroids in vivo. Br J Cancer. 2005;94:101-7.

109. Mantovani A, Sozzani S, Locati M, et al. Macrophage polarization: tumor-associated macrophages as a paradigm for polarized M2 mononuclear phagocytes. Trends Immunol. 2002;23:549-55.

110. Wu H, Xu J-B, He Y-L, et al. Tumor-associated macrophages promote angiogenesis and lymphangiogenesis of gastric cancer. J Surg Oncol. 2012;106:462-8.

111. Park J-Y, Sung J-Y, Lee J, et al. Polarized CD163 tumor-associated macrophages are associated with increased angiogenesis and CXCL12 expression in gastric cancer. Clin Res Hepatol Gastroenterol. 2016;40:357-65.

112. Kawahara A. Infiltration of thymidine phosphorylase-positive macrophages is closely associated with tumor angiogenesis and survival in intestinal type gastric cancer. Oncol Rep. 2010;24:405-15.

113. Sammarco G, Varricchi G, Ferraro V, et al. Mast cells, angiogenesis and lymphangiogenesis in human gastric cancer. Int J Mol Sci. 2019;20:2106.

114. Hiromatsu Y, Toda S. Mast cells and angiogenesis. Microsc Res Tech. 2003;60:64-9.

115. Kondo K, Muramatsu M, Okamoto Y, et al. Expression of chymasepositive cells in gastric cancer and its correlation with the angiogenesis. J Surg Oncol. 2005;93:36-42.

116. Mao X-Y. COX-2 expression in gastric cancer and its relationship with angiogenesis using tissue microarray. World J Gastroenterol. 2007;13:3466.

117. Tatsuguchi A, Matsui K, Shinji Y, et al. Cyclooxygenase-2 expression correlates with angiogenesis and apoptosis in gastric cancer tissue. Hum Pathol. 2004;35:488-95.
118. Joo Y-E, Rew J-S, Seo Y-H, et al. Cyclooxygenase-2 overexpression correlates with vascular endothelial growth factor expression and tumor angiogenesis in gastric cancer. J Clin Gastroenterol. 2003;37:28-33.

119. Wu Y-L, Fu S-L, Zhang Y-P, et al. Cyclooxygenase-2 inhibitors suppress angiogenesis and growth of gastric cancer xenografts. Biomedicine Pharmacotherapy. 2005;59(Suppl 2):S289-92.

120. Fu S-L. Anti-cancer effects of COX-2 inhibitors and their correlation with angiogenesis and invasion in gastric cancer. World $\mathrm{J}$ Gastroenterol. 2004;10:1971.

121. Chen C-N, Hsieh F-J, Cheng Y-M, et al. Expression of inducible nitric oxide synthase and cyclooxygenase- 2 in angiogenesis and clinical outcome of human gastric cancer. J Surg Oncol. 2006;94:226-33.

122. Zhang W, He X-J, Ma Y-Y, et al. Inducible nitric oxide synthase expression correlates with angiogenesis, lymphangiogenesis, and poor prognosis in gastric cancer patients. Hum Pathol. 2011;42:1275-82.

123. Hara K, Fukui H, Sun C, et al. Effect of REG I $\alpha$ protein on angiogenesis in gastric cancer tissues. Oncol Rep. 2015;33:2183-9.

124. Koide N, Nishio A, Igarashi J, et al. alpha-Fetoprotein-producing gastric cancer: histochemical analysis of cell proliferation, apoptosis, and angiogenesis. Am J Gastroenterol. 1999;94:1658-63.

125. Chen E, Tang C, Peng K, et al. ANGPTL6-mediated angiogenesis promotes alpha fetoproteinproducing gastric cancer progression. Pathol Res Pract. 2019;215:152454.

126. Zang M, Zhang Y, Zhang B, et al. CEACAM6 promotes tumor angiogenesis and vasculogenic mimicry in gastric cancer via FAK signaling. Biochim Biophys Acta. 2015;1852:1020-8.

127. Falco SD. The discovery of placenta growth factor and its biological activity. Exp Mol Med. 2012;44:1.

128. Lee G-H, Joo Y-E, Koh Y-S, et al. Expression of survivin in gastric cancer and its relationship with tumor angiogenesis. Eur J Gastroenterol Hepatol. 2006;18:957-63.

129. Li Q, Zhang N, Jia Z, et al. Critical role and regulation of transcription factor FoxM1 in human gastric cancer angiogenesis and progression. Cancer Res. 2009;69:3501-9.

130. Wang W-F. Krüppel-like factor 8 overexpression is correlated with angiogenesis and poor prognosis in gastric cancer. World J Gastroenterol. 2013;19:4309.

131. Tao X, Cheng L, Li Y, et al. Expression of CRYAB with the angiogenesis and poor prognosis for human gastric cancer. Medicine. 2019;98:e17799.

132. Zhou Y-J. Inactivation of PTEN is associated with increased angiogenesis and VEGF overexpression in gastric cancer. World J Gastroenterol. 2004;10:3225.

133. Li Y, Wu Z, Yuan J, et al. Long non-coding RNA MALAT1 promotes gastric cancer tumorigenicity and metastasis by regulating vasculogenic mimicry and angiogenesis. Cancer Lett. 2017;395:31-44.

134. Ma J, Sawai H, Matsuo Y, et al. Interleukin- $1 \alpha$ enhances angiogenesis and is associated with liver metastatic potential in human gastric cancer cell lines. J Surg Res. 2008;148:197-204.

135. Chung HW, Lim JB. High- mobility group box-1 contributes tumor angiogenesis under interleukin-8 mediation during gastric cancer progression. Cancer Sci. 2017;108:1594-601.

136. Wu X, Yang T, Liu X, et al. IL-17 promotes tumor angiogenesis through Stat3 pathway mediated upregulation of VEGF in gastric cancer. Tumor Biol. 2015;37:5493-501.

137. Yan W-F, Nie C-F, Wu G, et al. Soluble interleukin-2 receptor as a factor associated with angiogenesis in gastric cancer. Mol Med Rep. 2017;16:6916-9.

138. Wu Z-H, Lin C, Liu C-C, et al. MiR-616-3p promotes angiogenesis and EMT in gastric cancer via the PTEN/AKT/mTOR pathway. Biochem Biophys Res Commun. 2018;501:1068-73. 
139. Lee SH, Jung YD, Choi YS, et al. Targeting of RUNX3 by miR-130a and miR-495 cooperatively increases cell proliferation and tumor angiogenesis in gastric cancer cells. Oncotarget. 2015;6:33269-78.

140. Dai J, Wang J, Yang L, et al. miR-125a regulates angiogenesis of gastric cancer by targeting vascular endothelial growth factor $\mathrm{A}$. Int J Oncol. 2015;47:1801-10.

141. Chen H, Li L, Wang S, et al. Reduced miR-126 expression facilitates angiogenesis of gastric cancer through its regulation on VEGF-A. Oncotarget. 2014;5:11873-85.

142. Bai M, Li J, Yang H, et al. miR-135b delivered by gastric tumor exosomes inhibits FOXO1 expression in endothelial cells and promotes angiogenesis. Mol Ther. 2019;27:1772-83.
143. Zhang X, Dong J, He Y, et al. miR-218 inhibited tumor angiogenesis by targeting ROBO1 in gastric cancer. Gene. 2017;615:42-9.

Publisher's Note Springer Nature remains neutral with regard to jurisdictional claims in published maps and institutional affiliations. 